\title{
Intra-atrial tumour thrombus secondary to hepatocellular carcinoma
}

\section{Hetal Pandya, ${ }^{1}$ Chaitri Shah, ${ }^{2}$ Jitendra Lakhani, ${ }^{3}$ Micky Patel ${ }^{4}$}

1. Professor, 2. Associate Professor, 3. Professor and Head, 4. Junior Resident

\section{S.B.K.S. Medical Institute and Research Centre, Vadodara, Gujarat, India}

\section{CASE REPORT}

Please cite this paper as: Pandya $\mathrm{H}$, Shah C, Lakhani J, Patel

M. Intra-atrial tumour thrombus secondary to hepatocellular carcinoma AMJ 2013, 6, 6, 321-324. http://doi.org/10.21767/AMJ.2013.1635

\section{Corresponding Author:}

Hetal Pandya

Dept of Gen. Medicine, Dhiraj Hospital,

At \& PO Piparia,

District: Vadodara

Gujarat, India

Email: drhetalpandya@gmail.com

\section{Abstract}

Hepatocellular carcinoma ( $\mathrm{HCC}$ ) is one of the leading causes of cancer-related mortality. Hepatitis B \& C accounts for most HCC occurrences. It is very rare to see a tumour thrombus extending into the right atrium of the heart as a result of the invasion of HCC. This complication has a very poor prognosis as mean survival time is about three to four months. We encountered such a rare case of hepatocellular carcinoma having extensive tumour thrombus extending into the right atrium without any cardio respiratory distress or clinical finding suggestive of cardiovascular involvement. Key Words:

Hepatocellular carcinoma; tumour thrombus

\section{Implications for Practice}

1. What is known about this subject?

Hepatocellular carcinoma (HCC) is the fifth most common cancer in the world and an extension of tumour thrombus in the right atrium of the heart has been reported in $0.5-4 \%$ of HCC cases.

2. What is the key finding in this case report?

Asymptomatic presentation of extensive tumour thrombus extending from left portal vein, inferior vena cava to right atrium valve of heart at the time of diagnosis of HCC, an extremely rare situation which has very poor prognosis.
3. What are the implications for future practice? Early diagnosis of this fatal complication can prevent inappropriate use of resources.

\section{Background}

Worldwide, hepatocellular carcinoma (HCC) is the fifth most common cancer and the third most common cause of cancer-related death. ${ }^{1} \mathrm{HCC}$ accounts for $1-2.5 \%$ of all cancer in America. More than $80 \%$ of HCC worldwide is attributable to the combined effects of chronic hepatitis B \& C infections. $^{2,3}$ Many paraneoplastic manifestations are documented with HCC. Yet advanced HCC with an invasion of the inferior vena cava is an uncommon way for the disease to progress and it is rare to see an extension of tumour thrombus in the right atrium of the heart. ${ }^{4-6}$ Vallakati et al have reported such extension in 1-4\% of HCC cases. ${ }^{5}$ We report this relatively rare case of $\mathrm{HCC}$ with a complication of the inferior vena cava (IVC) tumour thrombus extending into right atrium.

\section{Case details}

A 55-year-old male with history of chronic alcohol intake, presented with right upper abdominal pain, which had been present for more than three months. It was associated with low grade fever and weight loss. He did not have any prior history of surgery, blood transfusions or jaundice. At the time of presentation, his vital signs were normal and he did not have pallor, icterus or pedal oedema. On examination, moderate hepatomegaly was noted. The rest of the physical examination was unremarkable. Complete blood count, basic biochemistry investigations and coagulation studies were within normal limits. ESR was elevated $(84 \mathrm{~mm} / \mathrm{hr}$ ). Liver function tests revealed total bilirubin of $1.8 \mathrm{mg} / \mathrm{dl}(\mathrm{N}$ : 0.2-1.0 mg/dl), direct bilirubin $0.8 \mathrm{mg} / \mathrm{dl}(\mathrm{N}: 0.1-0.4 \mathrm{mg} / \mathrm{dl})$, alanine aminotransferase (ALT) $82 \mathrm{IU} / \mathrm{L}(\mathrm{N}$ : up to $40 \mathrm{IU} / \mathrm{L}$ ), aspartate aminotransferase (AST) $118 \mathrm{IU} / \mathrm{L}(\mathrm{N}$ : up to $40 \mathrm{IU} / \mathrm{L}$ ) and alkaline phosphatase $40 \mathrm{IU} / \mathrm{L}$ (N: 80-290 IU/L). The patient was found to be HBsAg positive. $\alpha$-Fetoprotein (AFP) was significantly elevated $(>400 \mathrm{mg} / \mathrm{ml}, \mathrm{N}:<10 \mathrm{mg} / \mathrm{ml}$ ). An abdominal ultrasound showed a multicentre mass lesion in the left lobe of liver, another small lesion in the right lobe, metastatic paracaval lymphadenopathy and thrombus extending from the left portal vein branch, inferior vena 
cava to the right atrium of the heart. (Figures 1-2) Computed tomography (CT) of the abdomen further confirmed the ultrasonography findings. CT demonstrated multiple hypo dense infiltrative lesions with peripheral marked enhancement with neo-vascularisation from branches of the hepatic artery. There was also a thread- and streak-like pattern in the proximal left portal vein, which indicated malignant thrombus with a few enlarged necrotic lymph nodes. (Figures 3-4)

Figure 1: Ultrasonography showing large irregular hypoecohic lesion with internal heterogeneous area (necrosis \& fibrosis), mass seen in the left lobe of the liver and dilated IVC with the thrombosis in it

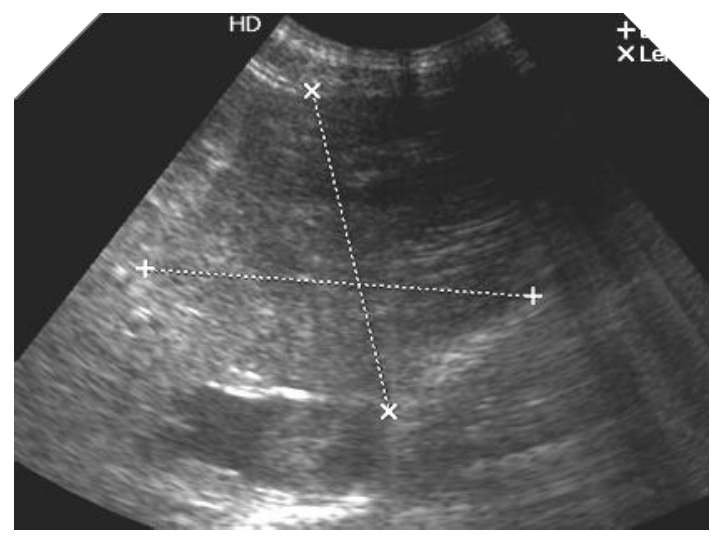

Figure 2: Dilatation of the IVC \& enlargement of the paracaval nodes on USG abdomen

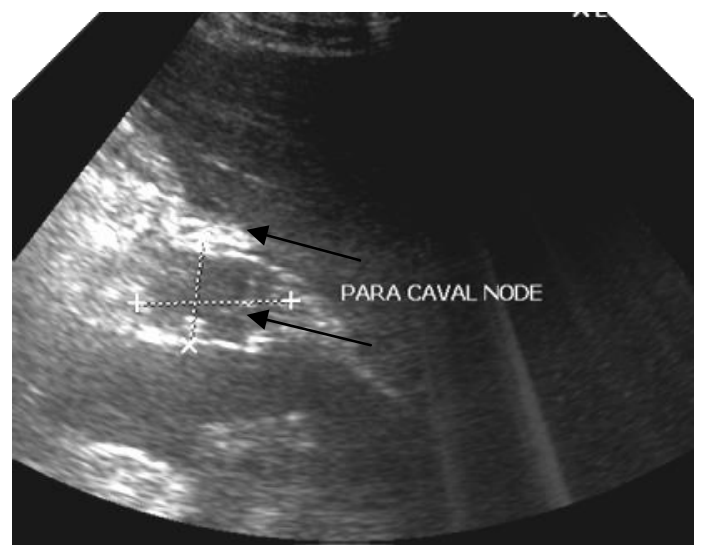

Figure 3: Thrombosis in the inferior vena cava with metastasis in the right lobe of liver with ascites on CT abdomen

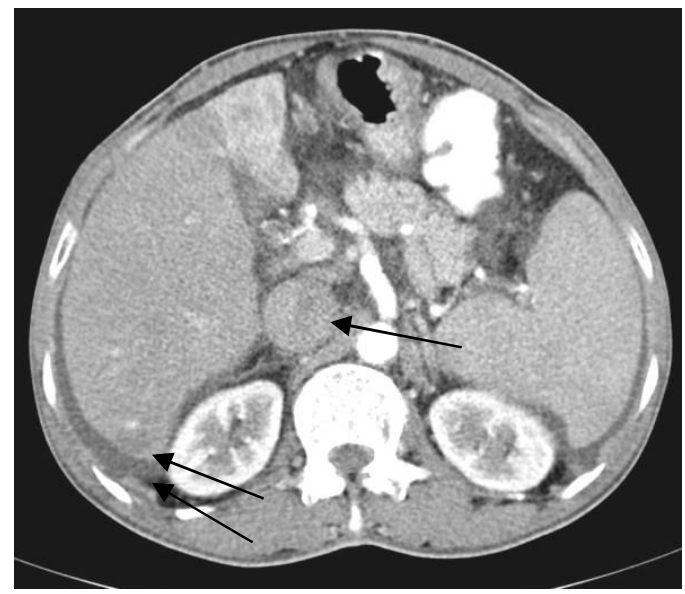

Figure 4: Tumour thrombus in the right atrium on CT of the chest

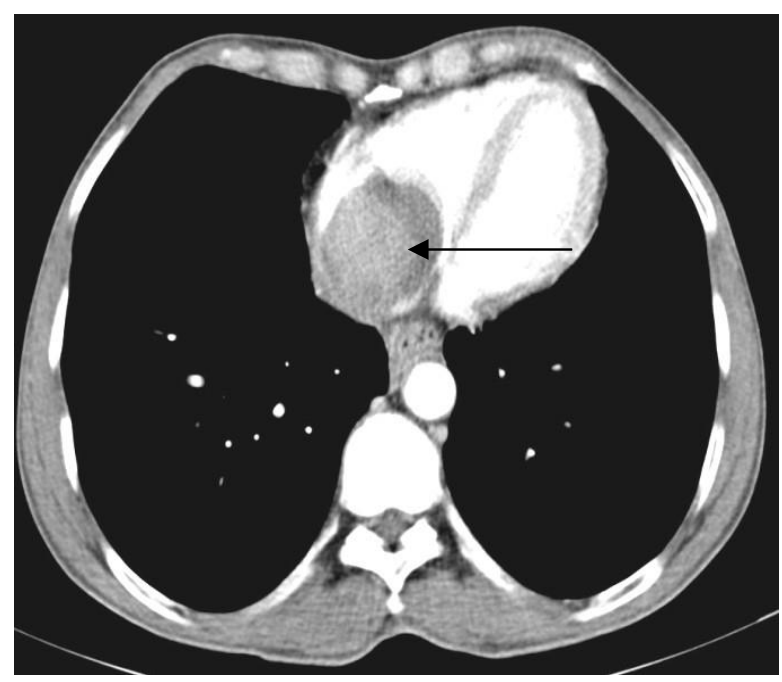

An ultrasound guided liver biopsy was done for further confirmation of diagnosis and staging of HCC. Microscopic features seen were moderate to severe degree of pheomorphism with multinucleation; hyperchromatism and increased mitosis associated with focal cholestasis. All features were suggestive of hepatocellular carcinoma (Figure 5). Transthoracic 2-D echocardiography showed a thrombus in the right atrium with mild dilatation of the chamber (Figure 6). The patient was referred to the cancer institute for further management. The final diagnosis made was stage IIIC HCC (according to the American Joint Committee on Cancer - AJCC) with malignant thrombus extending through the IVC up to the right atrium in a hepatitis B positive patient. 
Figure 5: Histopathological features suggestive of hepatocellular carcinoma

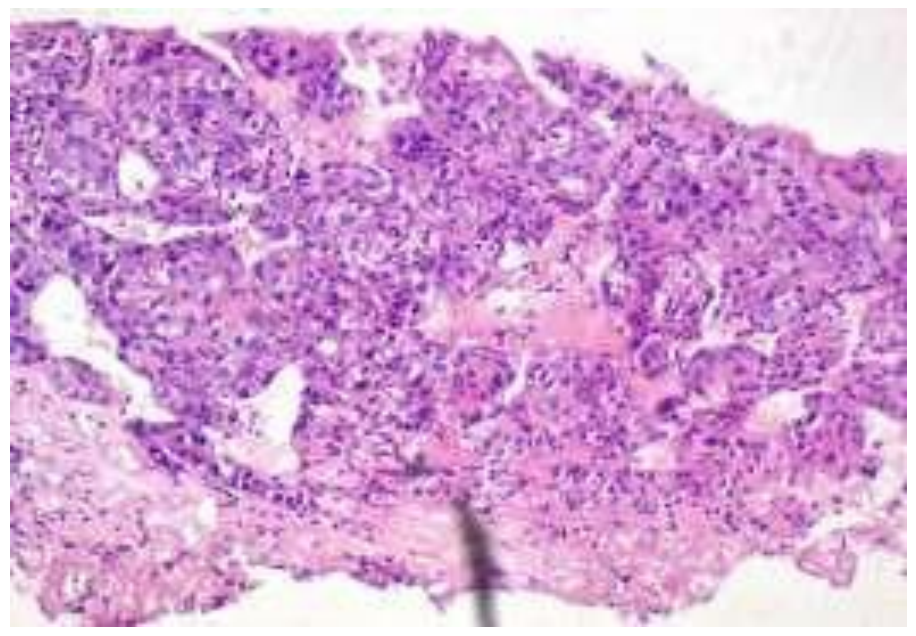

Figure 6: Echocardiography showing tumor thrombus in the right atrium of heart

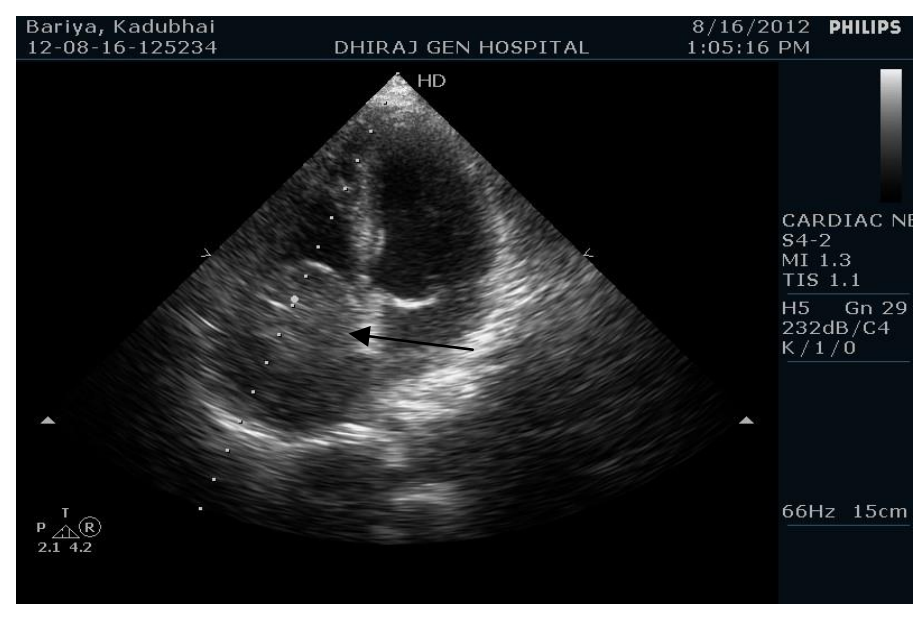

\section{Discussion}

Worldwide, HCC is the third most common cause of cancerrelated mortality and hepatitis $B$ is considered as one of the leading cause for the HCC. HCC develops from chronic hepatitis $B$ at the rate of $0.5 \%$ per year. ${ }^{1}$ Our patient also had hepatitis $B$ as the primary aetiology for development of HCC. We were unable to elicit a risk factor for hepatitis $B$ from the patient which is a common scenario among patients from South Asia.

The most common paraneoplastic syndromes reported in HCC are biochemical abnormalities without associated clinical consequences. Tumour thrombus is a very rare complication observed with HCC. According to Kim et al, only $0.53 \%$ of HCC patients have invasion to the IVC. ${ }^{7}$ An intra-atrial tumour thrombus is extremely rare in cases of $\mathrm{HCC}$ and is more commonly seen with malignancies of the kidney and adrenal glands. ${ }^{7}$ Our patient presented with a huge tumour thrombus extending from the left portal vein, the IVC to the right atrium. Such an extensive thrombus on presentation is extremely rare.

Extension of the tumour thrombus to the IVC and/or right atrium is usually first disclosed at autopsy; there are only a few reported cases in which the intra-atrial growth could have been diagnosed by non-invasive radiology and echocardiography prior to death. ${ }^{4,5}$ Our patient was admitted with complaints of right upper abdominal pain, low grade fever and weight loss. He had no cardiorespiratory findings despite having such a large thrombus. The literature also states that right atrial thrombus may not cause any symptoms, but can sometimes lead to shock from ball valve obstruction of the tricuspid valve, right heart failure, pulmonary emboli and sudden death. ${ }^{8,9}$

The tumour thrombus and its extension was diagnosed on ultrasonography of abdomen and echocardiography and confirmed by CT in the reported case. Echocardiography has been shown to be a useful additional diagnostic step for detection of cardiac metastasis and other complications like thrombosis. Echocardiography can provide information not only about the size and extension of the thrombus, but also about the mobility of the tumour thrombus and the relation of the valve and cardiac muscle with respect to the thrombus. With the invention of better non-invasive diagnostic approaches such as CT, MRI, and trans-thoracic echocardiography (TTE), it is very easy to diagnose many such rare and deep-seated complications in the early stages even before they manifest as was the case with our patient.

Surgical resection, liver transplantation, non-surgical procedures such as radio frequency ablation, intra-arterial chemoembolisation and drugs such as thalidomide and sorafenib (multikinase inhibitor of the platelet derived growth factor) have been shown to be effective for relieving symptoms and prolonging life in HCC patients with multiple or extensive metastasis. However, very high mortality rates are observed for advanced HCC with IVC and intra-atrial tumour thrombus extension. Mean survival time reported is three to four months whether the patient is treated or not. ${ }^{7}$ Hence aggressive therapy in patients with this rare and highly fatal complication is of little benefit due to their imminent, inevitable mortality. As many of our patients belong to lower socio-economical classes, screening for such asymptomatic but lethal complication can prevent inappropriate resource use and also can reduce discomfort to the patient of prolonged hospitalisation and aggressive treatment. Yet further data is required to generate more information on the balance of cost versus benefit for screening for this rare and fatal complication and what effect it would have on outcome. This information is 
important given the social and financial implications for often resource poor, affected, persons.

\section{Conclusion}

HCC patients are increasing in alarming numbers in the South-Asian subcontinent because of the increasing incidence of viral hepatitis $B$ and $C$ as well as alcoholinduced cirrhosis. We must remain vigilant about various uncommon manifestations associated with this carcinoma in our clinical practice which may be asymptomatic, as with our patient of HCC having intra-atrial tumour thrombus, this may influence the most appropriate treatment strategy.

\section{References}

1. Bosch FX, Ribes J, Borras J. Epidemiology of primary liver cancer. Semin Liver Dis. 1999;19(3):271-85.

2. Amin J, Dore GJ, O'Connell D L, Barlett M, Tracey E. Cancer incidence in people with hepatitis B or C infection: a large community-based linkage study. J Hepatol. 2006 Aug;45(2):197-203.

3. Chu CM. Natural history of chronic hepatitis B virus infection in adults with emphasis on the occurrence of cirrhosis and Hepatocellular carcinoma. J Gastroenterol Hepatol. 2000 May;15 Suppl:E25-30.

4. Masahiro N, Masue M, Hirofumi Y, Setsu S. A case of an intra-atrial tumour thrombus from hepatocellular carcinoma (HCC), first indicated by 67 Ga-citrate scintigraphy. Ann Nucl Med 1995; 9(1): 39-42.

5. Ajay V, Preeti C, Robort F, Jacob S. Intra-atrial tumour thrombi secondary to hepatoellular carcinoma responding to chemotherapy. N American Jr of Med Sci 2011; 3(9): 435437.

6. Hanada K, Fukui H, Tamura M, Tsujii T. An autopsy case of encapsulated hepatocellur carcinoma with its metastasis into right atrium and pericardium. Kan Tan Sui 1986; 12: 471-475.

7. Kim SU, Kim YR, Kim do Y, Kim JK, Lee HW, Kim BK, Han $\mathrm{KH}$, Chon $\mathrm{CY}$, Moon YM, Ahn SH. Clinical features and treatment outcome of advanced Hepatocellularcarcinoma with inferior vena caval invasion or atrial tumor thrombus. Korean J Hepatol. 2007 Sep;13(3):387-95.

8. Pellicelli AM, Barba J, Gomez AJ, Borgia MC. Echocardiographic follow up of right atrial tumoral invasion by hepatocarcinoma: a case report. Cardiologia. 1992 Feb;37(2):151-3.

9. Baca-Lopez FM, Ramirez-Arias E, Rayas-Gomez AL. Hepatocellular carcinoma with invasion into right cardiac cavities: Report of a case and literature review. J Am Soc Echocardiogr 2004; 17: 192-194.

\section{PEER REVIEW}

Not commissioned. Externally peer reviewed.

\section{CONFLICTS OF INTEREST}

The authors declare that they have no competing interests.

\section{PATIENT CONSENT}

The authors, Hetal Pandya, Chaitri Shah, Jitendra Lakhani, Micky Patel declare that:

1. They have obtained written, informed consent for the publication of the details relating to the patient in this report.

2. All possible steps have been taken to safeguard the patient's identity.

3. This submission is compliant with the requirements of institutional ethics committee. 